\title{
Approval of Itolizumab for COVID-19: A Premature Decision or Need of The Hour?
}

\author{
Shubham Atal ${ }^{1} \cdot$ Zeenat Fatima $^{1}$ (]) $\cdot$ Sadasivam Balakrishnan ${ }^{1}$
}

Published online: 13 October 2020

(c) Springer Nature Switzerland AG 2020

\begin{abstract}
Itolizumab is a first-in-class anti-CD6 monoclonal antibody that was initially developed for various cancers and was later developed and approved in India for treatment of moderate to severe chronic plaque psoriasis in 2013. This drug is now being re-purposed for COVID-19. The potential utility of itolizumab in COVID-19, based on its unique mechanism of action in ameliorating cytokine release syndrome (CRS), was proposed first in Cuba with approval of a single-arm clinical trial and expanded access use. Subsequently, a phase II, open-label, randomized, placebo-controlled trial has been conducted in 30 COVID-19 patients in India after receiving regulatory permission. Based on the results, the Indian drug regulatory agency recently approved itolizumab in July 2020 for 'restricted emergency use' for the treatment of CRS in moderate to severe acute respiratory distress syndrome (ARDS) due to COVID-19. This has drawn sharp criticism within the scientific community, with the approval being granted on the basis of a relatively small phase II trial, without conduct of a conventional phase III trial, and lacking availability of the claimed supportive real-world evidence in the public domain to date. In a global scenario where finding a successful treatment for COVID-19 is of utmost priority, a biologic agent has been re-purposed and approved with a successfully completed RCT, in a country where cases and mortality due to COVID-19 are growing exponentially. However, instead of welcoming the approval with open arms, many doubts are being raised. This is an issue that needs to be considered and dealt with sensitively, as well as scientifically.
\end{abstract}

\section{Key Points}

Itolizumab is a drug originally used for treating psoriasis that has been recently approved for treating complications in moderate to severe COVID-19 patients in India and Cuba.

Itolizumab is an anti-CD6 humanized monoclonal antibody that suppresses $\mathrm{T}$ cell activation and downregulates the synthesis of proinflammatory cytokines and adhesion molecules.

While approval for restricted emergency use of this agent makes a potentially life-saving treatment available for an important subset of COVID patients, the relative paucity of available evidence requires thorough analysis and publication, along with further phase III or real-world investigations.

Zeenat Fatima

zeenat.sr2018@aiimsbhopal.edu.in

1 Department of Pharmacology, All India Institute of Medical Sciences Bhopal, 3rd Floor, Medical College Building, Saket Nagar, Bhopal 462020, India

\section{Introduction}

Amidst the ongoing struggle to find a successful treatment for COVID-19, itolizumab became the first novel biologic therapy to be approved in the world for COVID-19-for patients with moderate to severe complications. The Indian drug regulatory agency (Central Drug Standard Control Organisation, CDSCO) recently approved itolizumab for 'restricted emergency use' for treatment of cytokine release syndrome (CRS) in moderate to severe acute respiratory distress syndrome (ARDS) due to COVID-19, followed by a similar approval in Cuba [1,2]. 'Restricted emergency use' requires fulfilment of certain requirements for use including written informed consent of patients, explaining risks and benefits of the drug and putting a risk management plan in place to manage any adverse effects. The drug can be given only in hospitals and medical institutions, and is not meant for sale at retail pharmacies [3]. Itolizumab is a first-in-class monoclonal antibody, first developed for auto-immune diseases in India, where it received approval for treatment of patients with active moderate to severe chronic plaque psoriasis in 2013 [4]. It 
has been approved for the same indication in Cuba as well, but not in other countries yet [5].

The potential utility of itolizumab in COVID-19, based on its mechanism of action, led to expanded access use and conduct of a trial in Cuba, retrospectively registered with the Cuban regulatory agency (Center for the State Control of Medicines, Equipment and Medical Devices, CECMED) in May 2020. This trial recruited patients with critical and severe illness, and moderately ill patients with very high risk of developing severe symptoms [6]. Encouraged by the initial promising results with the first few patients seen in this index trial, regulatory permission was received to repurpose the drug for COVID-19 in India. The drug was studied in an open-label, randomized, controlled, phase II trial at four hospitals in India to assess the safety and efficacy of itolizumab in preventing CRS in moderate to severe ARDS due to COVID-19 [7]. On the basis of the results of this phase II trial, itolizumab was granted approval for 'restricted emergency use' for this indication in COVID-19 patients in July 2020 [1]. The data obtained from the Cuban single-arm trial, in conjunction with the Indian data, also led to the drug's approval for the same indication in COVID-19 in Cuba later in the same month; data on the results submitted to the Cuban authority are not available [2]. On one hand, this is welcome news as a potentially life-saving treatment is now available for an important subset of COVID-19 patients-those having CRS-mediated ARDS. But, on the other hand, the relative paucity of evidence available makes it necessary to carefully evaluate the scientific validity of this approval. Further questions arise as the drug has not been included in the clinical management protocol recommended by the national task force on COVID-19 in India, despite getting approval from the country's regulator, because of insufficient evidence. The innovator company, Biocon Ltd, claims to have supportive real-world data on nearly 1000 patients that they intend to provide to the authorities concerned [8].

\section{Itolizumab: The Molecule, Mechanism of Action, and Safety}

Itolizumab is a humanized anti-CD6 monoclonal antibody $(\mathrm{mAb})$, specifically targeting the SRCR-1 (scavenger receptor cysteine-rich like domain 1) of CD6. It has a molecular weight of $148 \mathrm{kDa}$ and contains two heavy chains and two light chains linked with a disulfide bond [9-11]. The parent antibody of itolizumab is ior T1, a murine $\mathrm{mAb}$, which has shown therapeutic efficacy in psoriasis and rheumatoid arthritis. Itolizumab, being a humanized $\mathrm{mAb}$, is less immunogenic and has a better safety profile with the same therapeutic efficacy as ior T1 $[10,12]$.

\subsection{Mechanism of Action}

Itolizumab is a $\mathrm{mAb}$ directed against $\mathrm{CD}$, a surface glycoprotein found on mature $T$ cells, immature $B$ cells, the $B 1 \mathrm{a}$ subset of B lymphocytes, and certain regions of the brain. It plays a pivotal role in cell proliferation, adhesion, differentiation, and survival. Its extracellular region is composed of three SRCR domains. CD6 is a co-stimulatory molecule, and its stimulation is responsible for $\mathrm{T}_{\mathrm{h}} 1$ activation, and differentiation of $\mathrm{T}$ cells, which promotes a proinflammatory response. Thus, by inhibiting CD6, itolizumab downregulates the synthesis of proinflammatory cytokines and adhesion molecules that eventually leads to reduced interferon- $\gamma$ (IFN $\gamma$ ), interleukin (IL)-6, and tumor necrosis factor- $\alpha$ $(\mathrm{TNF} \alpha)$ levels, along with reduced T-cell infiltration at the inflammatory sites [4].

Interestingly, these anti-inflammatory effects are not achieved by inhibiting ligand binding and causing T-cell depletion, but by inhibiting new receptor formation, stimulating loss of existing receptors or by blockade and internalization or downregulation of receptors. In vitro experiments have shown that itolizumab does not cause T-cell depletion but inhibits T-cell proliferation induced in the presence of ALCAM (activated leukocyte cell adhesion molecule or CD 166) and excess IL-2, and downregulates the phosphorylation of intracellular proteins implicated in the CD6-mediated activation pathways [4]. So, it inhibits the downstream inflammatory cascade by a unique mechanism of action.

\subsection{Safety and Monitoring}

Safety data for itolizumab has been derived mainly from the two multicenter clinical trials of patients with chronic plaque psoriasis, and from a study of patients with rheumatoid arthritis $[10,13,14]$. No treatment-related severe adverse effects (AEs) were noted in these trials. The various AEs observed with itolizumab were infusion-related reactions (such as nausea, rash, urticaria, flushing, cough, wheezing, dyspnea, dizziness, and headache), pyrexia due to infections, pruritus, antidrug antibody formation, and transient decrease in mean lymphocyte count. Infusion reactions, the most common adverse event, have been reported in $12-15 \%$ of patients. The frequency and severity of infusion reactions were noted to decrease with subsequent infusions. Other frequent adverse events ( $>5 \%)$ are diarrhea, pyrexia, upper respiratory infections, and pruritus $[4,15]$.

Itolizumab is not recommended for patients with active infection or a history of recurring infections, latent TB 
infection, children, patients with hepatic and renal impairment, or pregnant and lactating women $[4,11]$.

\section{Rationale for Use in Cytokine Release Syndrome (CRS)-Induced Acute Respiratory Distress Syndrome (ARDS) due to COVID-19}

As summarized in our article, CRS is a phenomenon implicated in severe COVID-19 disease where there is an uncontrolled release of inflammatory cytokines including various interleukins, TNF $\alpha$, IFN $\gamma$, and other mediators [16]. This severe systemic acute inflammatory response can cause damage to multiple organs including acute lung injury and ARDS, which is being reported as one of the serious complications in COVID-19 patients [17, 18]. Higher levels of these cytokines and mediators have been observed in the plasma of COVID-19 patients requiring intensive care [19]. A 2.9-fold higher mean IL-6 concentration has been shown in patients with complicated COVID-19 compared with noncomplicated disease [20].

Amelioration of CRS is an important strategy to reduce the severity of pulmonary and systemic complications in such COVID-19 patients, which can reduce the need for intensive care and mechanical ventilation. Based on the strategy of reducing the pulmonary hyperinflammation through modulating the CRS, several randomized controlled trials (RCTs) of IL-6 inhibitors such as tocilizumab and sarilumab are exploring their potential in patients with serious COVID-19 across the globe [16].

The mechanism of action of itolizumab is different from that of other selective immunomodulatory approaches (e.g., IL inhibitors) being tried for treatment of CRS associated with COVID-19, as it selectively targets the CD6-ALCAM pathway and decreases the activation, proliferation as well as differentiation of $\mathrm{T}$ cells into pathogenic effector $\mathrm{T}$ cells. Production of pro-inflammatory cytokines like IL-6, TNF $\alpha$, and INF $\gamma$ is decreased along with T-cell infiltration, but the regulatory function of Treg cells is preserved [9-14]. It may perhaps be a better immunomodulatory approach for combating CRS-induced ARDS in COVID-19 with its ability to stop the production of pro-inflammatory cytokines rather than just inhibiting these cytokines as the other agents do.

\section{Clinical Trials of Itolizumab for the Treatment of COVID-19}

A phase II, multi-centric, open-label, two-arm, randomized, pivotal clinical trial was conducted in 30 patients in India [21]. The trial included adults aged $>18$ years of either sex with a confirmed virological diagnosis of SARS-CoV-2 infection with reverse transcription polymerase chain reaction (RT-PCR) assay who were hospitalized due to clinical worsening of COVID-19 infection with an oxygen saturation at rest in ambient air $\leq 94 \%$. Patients had moderate to severe ARDS, as defined by a ratio of arterial oxygen partial pressure and fractional inspired oxygen $\left(\mathrm{PaO}_{2} / \mathrm{FiO}_{2}\right)$ of $<200$, or $>25 \%$ deterioration from the immediate previous value. In case of a delay in RT-PCR results, biomarker data-either baseline serum ferritin level $\geq 400 \mathrm{ng} / \mathrm{mL}$ or IL-6 levels $>4$ times the upper limit of normal (ULN) -was required for inclusion of the patient into the study. Patients with known severe allergic reactions to mAbs; known history of hepatitis B, hepatitis C, or HIV; active tuberculosis (TB) infection or having a history of inadequately treated or latent TB; absolute neutrophil count (ANC) $<1000 / \mathrm{mm}^{3}$, platelet count $<50,000 / \mathrm{mm}^{3}$, and absolute lymphocyte count (ALC) $<500 / \mathrm{mm}^{3}$; those on immunosuppressant drugs in the past 6 months; and those who had participated in other clinical drug trials using anti-IL-6 therapy were excluded [15].

Itolizumab was administered at a loading dose of $1.6 \mathrm{mg} /$ $\mathrm{kg}$, chosen as it is the approved dose in patients with chronic plaque psoriasis and has been administered as an intravenous (IV) infusion in several phase II and III clinical trials, without any evidence of dose-limiting toxicities. An additional dose of $0.8 \mathrm{mg} / \mathrm{kg}$ was administered after 1 week in some patients, if required, based on the physician's discretion; up to four weekly doses were allowed in the study [15].

With a 2:1 randomization, 20 patients were randomized to receive itolizumab plus supportive care, while 10 patients received supportive care alone in the control arm. The primary outcome was comparison of the 1-month mortality rate between the two arms. The secondary outcomes were plasma levels of biomarkers, lymphocyte count, C-reactive protein (CRP), $\mathrm{PaO}_{2} / \mathrm{FiO}_{2}$, radiological response, duration of hospitalization, and remission of respiratory symptoms [7,21]. All patients receiving itolizumab recovered fully and were discharged from hospital, whereas three out of ten patients (30\%) in the control arm died. There was also significant improvement in key efficacy parameters of lung function such as $\mathrm{PaO}_{2}$ and $\mathrm{SpO}_{2}$ (oxygen saturation) without increasing oxygen flow in the itolizumab arm. All patients on itolizumab were weaned off oxygen by day 30 , and none needed ventilator support, unlike the control arm. Key secondary endpoints of the clinical markers of inflammation such as IL-6, TNF $\alpha$, serum ferritin, d-dimer, lactate dehydrogenase (LDH), and CRP showed clinically significant suppression after itolizumab dosing and correlated well with clinical and radiological improvement in symptoms and chest X-ray images, respectively. Itolizumab was well tolerated overall and found to be safe, with the infusion reactions manageable with slowing the infusion rate [21]. 
These results are in line with the findings of the Cuban study, a single-arm, non-controlled clinical trial, where 80 COVID-19 patients were treated with itolizumab [15, 21]. It has been claimed that $79 \%$ of severely ill patients were discharged from the intensive care unit (ICU) after 14 days of treatment, while moderately ill patients showed a reduction in the rate of disease progression. However, no further details about this trial are available. Two studies on preprint servers are available presently; both are from the same Cuban trial (RPCEC00000311). One study shows that a single dose of itolizumab decreased the serum IL-6 levels after $48 \mathrm{~h}$ of administration in 24 moderate to critically ill elderly COVID-19 patients [22]. In another study, the authors concluded that in 19 moderately ill elderly COVID-19 patients, itolizumab treatment was associated with a significantly reduced risk of admission to ICU and a 10 times lower risk of death [23].

\section{Approval for COVID-19 and Controversy}

The approval of itolizumab for restricted emergency use to treat COVID-19 patients with the complication of moderate to severe ARDS comes at a time when cases and hospitalizations are increasing alarmingly; the mortality has exceeded 80 thousand in India [24]. This approval presumably comes with the intention of providing a viable life-saving treatment option for a specific subset of COVID-19 patients. Itolizumab is not a new investigational drug, having been approved since 2013 for psoriasis. The drug is being repurposed and positioned as a relatively more affordable option for treating CRS in COVID-19 patients compared with other immunomodulatory drugs like tocilizumab, which are also being used off-label. Tocilizumab is still under investigation in various countries including India, at the stage of phase III trials $[16,25]$. There is not enough evidence yet from completed studies that these drugs reduce mortality in COVID-19 patients. Under these circumstances, the recent approval of itolizumab on the basis of a phase II trial has understandably drawn sharp scrutiny from experts. Questions have been raised, mainly regarding the small sample size of the trial, exemption of a larger phase III trial, lack of published data regarding off-label use for COVID-19, and substantial claims being made through media without peer review or scientific publication [26]. These arguments have been defended by the company mainly on the basis of the desperate need for treatment to prevent mortality due to complications such as ARDS in seriously ill COVID-19 patients amidst the burgeoning pandemic, and that their trial successfully fulfilled all scientific and regulatory requirements [21].

Inconsistencies and potential fallacies have been observed by the scientific community pertaining to the study design used and withdrawal of two patients who experienced adverse events on initiation who were not considered randomized and were excluded from the trial analysis; one of these patients later died [27]. Does this breach the principle of intention-to-treat analysis? Moreover, there is confusion whether all 30 patients were actually randomized as it was reportedly stated that the first five participants were given itolizumab sequentially. This was done as per recommendations of the trial's drug and safety monitoring board. It also purportedly led to refusal by the subject expert committee (SEC) of CDSCO to give the emergency approval to itolizumab at an earlier stage in late May 2020 [28, 29]. From the data made available to date, the baseline $\mathrm{SpO}_{2}$ and values of inflammatory markers like CRP, D-dimer, ferritin, and LDH are not known; instead, a difference in proportion of improvement or mean change from baseline has been provided [27]. While all patients were on oxygen at baseline, a higher proportion of patients (40\%) were on non-invasive ventilation (NIV) in the control arm at baseline compared with the treatment arm $(25 \%)$, three of whom eventually went on to have the adverse outcome of death [27].

\section{Discussion}

There is no doubt that finding a successful treatment for COVID-19 that can save lives in this pandemic, which has taken close to 0.9 million lives worldwide to date, is of utmost priority [30]. In this scenario, a biologic agent has been re-purposed, successfully completed a RCT, and received approval for restricted emergency use in a country where cases and mortality due to COVID-19 are growing exponentially. So, instead of welcoming the approval with open arms, why are so many doubts being raised? This is an issue that needs to be considered and dealt with sensitively, as well as scientifically.

The trial that led to itolizumab approval in India was an open-label, multicenter RCT, but the total sample size, being a phase II study, was only 30 patients, of whom only 20 patients actually received the drug [21]. Although the primary endpoint (i.e., mortality at 1 month) was significantly in favor of itolizumab, it cannot be claimed with certainty that this result would reflect real-world effectiveness. It is unclear whether a design such as Simon's two-stage design, which can lead to greater confidence in the significance of results obtained even from a smaller size phase II trial, was followed for this trial or not [31]. However, it is to be noted that this type of study design is suitable for a single-arm study.

While the sample size may have been sufficient for the primary outcome parameters, the trial is likely to be underpowered for many secondary efficacy parameters; the 
'statistically significant' advantage of itolizumab for these parameters does not hold credence. It is also not known what constituted the best standard of care at the sites in the trial or whether steroids were part of the treatment regimens for these patients. This is important in light of the established effectiveness of steroids in patients with moderate to severe Covid-19 pneumonia [32]. The results are certainly encouraging, and are claimed to have been obtained through fulfilment of the due regulatory requirements, leading the Indian drug regulator to approve the drug for emergency use. The need to conduct a phase III clinical trial has also been waived, allowing the innovator to carry out post-marketing surveillance (phase IV) [33]. This adds further to the scrutiny and raises more questions. Typically, phase II trials are designed to evaluate the drug's efficacy in people with the disease being studied along with determining the common short-term adverse effects and risks associated with the drug. They also test different dose regimens of the study molecule to derive the optimal dosage for disease. A phase III trial is a large, multi-center trial considered crucial to proving the efficacy of a drug in a sufficient number of patients compared with placebo or standard of treatment; it provides evidence of clinical and statistical significance of any treatment effects obtained [34]. These questions have perhaps compelled the regulator to instruct the innovator company to revise its proposed phase IV protocol to keep safety as the primary objective, and increase the sample size as well as geographic distribution of study sites [35]. Furthermore, media claims of mortality reduction in COVID-19 have been made while the results have not yet been peer reviewed and scrutinized within the scientific community. Whether the study was methodologically robust cannot be determined unless the study protocol or the results in their entirety are available. Now there are reports questioning the scientific validity of this phase II trial and pointing out inadequacies in its design [36]. The Cuban regulatory study is non-randomized and single arm (non-controlled); data available for that study is even more scant [37].

It was proclaimed earlier that the real-world data for 'off-label' use of the drug on compassionate grounds will be published; all patients being cured of COVID-19 [21]. The latest statement claims availability of real-world evidence on 1000 patients [38]. It is being argued that any such real-world data for itolizumab is less likely to include cases that recovered without the use of itolizumab, and it may include only those patients who were successfully treated with the drug, which will not reflect the true results [36]. The lack of confidence in the drug, or rather in the evidence available to date, has been echoed in the exclusion of the drug from the latest clinical management protocol released by the national task force for COVID-19 in India, and a statement made by the chief of the apex body of medical research in the country [39]. However, in Cuba it has been included in the COVID-19 management protocol since April, before the grant of approval [40].

Itolizumab, which is manufactured in India, is relatively cheaper than other lifesaving options in severe COVID19 , such as tocilizumab or remdesivir, and its randomized trial is being touted as 'robust'. However, these justifications have seemingly not been accepted by the scientific community. The approved itolizumab formulation of $25 \mathrm{mg} / 5 \mathrm{~mL}$ costs INR7950 ( US $\$ 105$ ) per vial. The average cost of therapy at a dose of $1.6 \mathrm{mg} / \mathrm{kg}$, comprising four vials, is estimated to be $\sim$ INR32,000 ( USD425), which is less than the treatment cost with tocilizumab, which varies between INR45,000 and INR50,000 ( USD600-670) per vial [41]. However, this cost is still high, as only a small proportion of patients in India can access or rather afford this cost for complete treatment where the average per capita monthly income is INR11,254 ( USD150) [42].

The data and protocols from the drug trials in India and Cuba should be published at the earliest in their entirety; this may assuage many of the doubts and concerns regarding the strength of evidence. Even with the restricted use approval, a phase III trial of the drug should have been directed in India, as for tocilizumab, which is currently undergoing a large phase III trial in the country despite more real-world and randomized data being available for this drug. However, the emergency due to this pandemic has to be considered; the approval given is not completethe restricted use necessitates fulfilment of important prerequisites of use. The phase II data and the claimed realworld evidence does come entirely from the respective countries of approval, which gives some confidence for the use of the drug.

\section{Conclusion}

At this point in time, a guarded approach is needed regarding both use and evaluation of itolizumab as a drug for treatment of COVID-19. Perhaps publication of the trial data from the Indian and Cuban studies with support from the real-world off-label use data may provide satisfactory answers to some, if not all, of the crucial questions raised. Even so, more evidence would undoubtedly be warranted in terms of both randomized and real-world studies to be really convinced of the efficacy and safety of this drug for its purported use in COVID-19. For now, itolizumab does give physicians a therapeutic option to potentially save lives in an important subset of COVID-19 patients. 


\section{Declarations}

Funding None.

\section{Conflicts of interest None.}

Ethics approval Not applicable.

Consent to participate Not applicable.

Consent for publication Not applicable.

Availability of data and material Not applicable.

Code availability Not applicable.

Authors' contributions All the authors have substantially contributed to the conception, and drafting the work and revising it critically for important intellectual content and final approval of the version to be published.

\section{References}

1. Ministry of Health and Family welfare [Press Release]. DCGI gives Nod for Restricted Emergency Use to Itolizumab for moderate to severe COVID-19 patients. https://pib.gov.in/PressRelea sePage.aspx?PRID=1637926. Accessed 17 Aug 2020.

2. Plenglish, 2020. Cuba authorizes the use of Itolizumab in cases of Covid-19. https://www.plenglish.com/index.php?o=rn\&id=58171 $\& \mathrm{SEO}=$ cuba-authorizes-the-use-of-itolizumab-in-cases-of-covid -19. Accessed 17 Aug 2020.

3. FDA.gov.in. Emergency use authorization. https://www.fda.gov/ emergency-preparedness-and-response/mcm-legal-regulatory -and-policy-framework/emergency-use-authorization. Accessed 17 Aug 2020.

4. Menon R, David BG. Itolizumab-a humanized anti-CD6 monoclonal antibody with a better side effects profile for the treatment of psoriasis. Clin Cosmet Investig Dermatol. 2015;8:215-22.

5. Budamakuntla L, Madaiah M, Sarvajnamurthy S, Kapanigowda $\mathrm{S}$. Itolizumab provides sustained remission in plaque psoriasis: a 5-year follow-up experience. Clin Exp Dermatol. 2015;40(2):152-5.

6. Saavedra D, Añé-Kourí AL, Sánchez N, Filgueira LM, Batancourt J, Herrera C, et al. An anti-CD6 monoclonal antibody (Itolizumab) reduces circulating IL-6 in severe COVID19 elderly patients. 04 June 2020, PREPRINT (Version 1). Research Square. https://doi.org/10.21203/rs.3.rs-32335/v1. Accessed 17 Aug 2020.

7. CTRI. CTRI/2020/05/024959. A multi-centre, open label, two arm randomized, pivotal phase 2 trial to study the efficacy and safety of itolizumab in COVID-19 complications. http://ctri.nic.in/Clini caltrials/showallp.php?mid $1=42878 \&$ EncHid=\&userName $=$ itoli zumab. Accessed 17 Aug 2020.

8. Livemint. Biocon to give more data on itolizumab after use in covid-19 treatment rejected. 2020. https://www.livemint.com/ news/india/biocon-to-give-more-data-on-itolizumab-after-use-incovid-19-treatment-rejected-11595858120238.html. Accessed 17 Aug 2020.

9. Aira LE, López-Requena A, Fuentes D, et al. Immunological and histological evaluation of clinical samples from psoriasis patients treated with anti-CD6 itolizumab. Mabs. 2014;6(3):783-93. https ://doi.org/10.4161/mabs.28376.
10. Rodriguez PC, Torres-Moya R, Reyes G, Molinero C, Prada D, Lopez AM, et al. A clinical exploratory study with itolizumab, an anti-CD6 monoclonal antibody, in patients with rheumatoid arthritis. Results Immunol. 2012;2:204-11. https://doi.org/10.1016/j. rinim.2012.11.001.

11. Alzumab (Itolizumab) solution for iv infusion [prescribing information]. India:Biocon, Inc; 2013. http://www.biocon.com/ docs/prescribing_information/immunotherapy/alzumab_pi.pdf. Accessed 17 Aug 2020.

12. Alonso-Ramirez R, Loisel S, Buors C, Pers JO, Montero E, Youinou P, Renaudineau Y. Rationale for targeting CD6 as a treatment for autoimmune diseases. Arthritis. 2010;2010:130646. https ://doi.org/10.1155/2010/130646.

13. Anand A, Assudani D, Nair P, Krishnamurathy S, Deodhar S, Arumugam M, et al. Safety, efficacy and pharmacokinetics of T1h, a humanized anti-CD6 monoclonal antibody, in moderate to severe chronic plaque psoriasis - results from a randomized phase II trial. J Immunol. 2010;184:96-103.

14. Krupashankar DS, Dogra S, Kura M, Saraswat A, Budamakuntala L, Sumathy TK, et al. Efficacy and safety of itolizumab, a novel anti-CD6 monoclonal antibody, in patients with moderate to severe chronic plaque psoriasis: results of a double-blind, randomized, placebo-controlled, phase-III study. J Am Acad Dermatol. 2014;71(3):484-92.

15. Loganathan $\mathrm{S}$, Athalye SN, Joshi SR. Itolizumab, an anti-CD6 monoclonal antibody, as a potential treatment for COVID-19 complications. Expert Opin Biol Ther. 2020;9:1025-31. https://doi. org/10.1080/14712598.2020.1798399.

16. Atal S, Fatima Z. IL-6 inhibitors in the treatment of serious COVID-19: a promising therapy? Pharm Med. 2020. https://doi. org/10.1007/s40290-020-00342-z.

17. Gu Y, Hsu AC, Pang Z, Pan H, Zuo X, Wang G, et al. Role of the innate cytokine storm induced by the influenza A virus. Viral Immunol. 2019;32:244-51. https://doi.org/10.1089/ vim.2019.0032.

18. Gibson PG, Qin L, Puah SH. COVID-19 acute respiratory distress syndrome (ARDS): clinical features and differences from typical pre-COVID-19 ARDS. Med. J. Aust. 2020;213:54-56.e1. https:// doi.org/10.5694/mja2.50674.

19. Huang C, Wang Y, Li X, Ren L, Zhao J, Hu Y, et al. Clinical features of patients infected with 2019 novel coronavirus in Wuhan. China Lancet. 2020;395:497-506.

20. Coomes EA, Haghbayan H. Interleukin-6 in COVID-19: a systematic review and meta-analysis. medRxiv. 2020. https://doi. org/10.1101/2020.03.30.20048058. Accessed 29 Apr 2020.

21. Biocon.com. Biocon Presented Insights into Clinical Study That Enabled DCGI Approval of Itolizumab for COVID-19. https:// www.biocon.com/biocon-presented-insights-into-clinical-study -that-enabled-dcgi-approval-of-itolizumab-for-covid19. Accessed 17 Sep 2020.

22. Saavedra D, Ane-Kouri AL, Sanchez N, Filgueira LM, Betancourt $\mathrm{J}$, herrera C, et al. An anti-CD6 Monoclonal antibody (itolizumab) reduces circulating IL-6 in severe covid-19 elderly patients, 04 June 2020, PREPRINT (Version 1) available at Research Square. https://doi.org/10.21203/rs.3.rs-32335/v1.

23. Ramos-Suzarte M, Diaz Y, Martin Y, Calderon NA, Santiago $\mathrm{W}$, Vinet $\mathrm{O}$, et al. Use of a humanized anti-CD6 monoclonal antibody (itolizumab) in elderly patients with moderate COVID-19. medRxiv 2020.07.24.20153833. https://doi. org/10.1101/2020.07.24.20153833.

24. Ministry of health and family welfare government of India. https ://www.mohfw.gov.in/. Accessed 17 Aug 2020.

25. CTRI. A study on treatment of COVID-19 patients with study drug along with standard of care. http://ctri.nic.in/Clinicaltrials/ pmaindet 2 .php?trialid $=43485 \&$ EncHid $=\&$ userName $=$ tocilizuma b. Accessed 17 Aug 2020. 
26. Biocon.com. Biocon's breakthrough drug itolizumab receives DCGI nod for its use in moderate to severe COVID-19 patients. https://www.biocon.com/docs/Biocon_PR_Itolizumab_Appro ved_for_Covid_India.pdf. Accessed 17 Aug 2020.

27. Itolizumab COVID19 Study Results. https://www.biocon.com/ docs/FINAL_Biocon_Itolizumab_COVID19_Study_Summa ry.pdf. Accessed 17 Sep 2020.

28. The Wire Science, 2020. The worrying ways in which Biocon's itolizumab trial was deeply flawed. https://science.thewire.in/thesciences/biocon-itolizumab-phase-ii-clinical-trial-cdsco-simon -two-stage-inconsistencies/. Accessed 21 Sep 2020.

29. Recommendations of the SEC meeting to examine COVID-19 related proposal under accelerated approval process made in its 78 th meeting held on 28.05.2020 at CDSCO, HQ New Delhi. https://cdsco.gov.in/opencms/opencms/system/modules/CDSCO .WEB/elements/common_download.jsp?num_id_pk=MTEwNQ. Accessed 21 Sep 2020.

30. COVID-19 Dashboard by the Center for Systems Science and Engineering (CSSE) at Johns Hopkins University (JHU). https:// coronavirus.jhu.edu/map.html. Accessed 17 Aug 2020.

31. Simon R. Optimal two-stage designs for phase II clinical trials. Control Clin Trials. 1989;10(1):1-10.

32. Omazini BM, Maia IS, Cavalcanti AB, Berwanger O, Rosa RG, Veiga VC, et al. Effect of dexamethasone on days alive and ventilator-free in patients with moderate or severe acute respiratory distress syndrome and COVID-19: the CoDEX randomized clinical trial. JAMA. 2020. https://doi.org/10.1001/jama.2020.17021.

33. Times of India, 2020. Key phase 3 clinical trial waived for Biocon drug. https://timesofindia.indiatimes.com/city/mumbai/key-phase -3-clinical-trial-waived-for-biocon-drug/articleshow/76948701. cms. Accessed 17 Aug 2020.

34. FDA. The drug development process. https://www.fda.gov/patie nts/drug-development-process/step-3-clinical-research. Accessed 17 Aug 2020.
35. The Hindu Business Line, 2020. Covid-19: Biocon to amend phase-4 trial protocol for psoriasis drug Itolizumab. https://www. thehindubusinessline.com/news/national/covid-19-biocon-toamend-phase-4-trial-protocol-for-psoriasis-drug-itolizumab/artic le32261837.ece. Accessed 17 Aug 2020.

36. Science The Wire, 2020. Itolizumab: why real-world data can't substitute randomised controlled trials. https://science.thewire.in/ author/jammi-nagaraj-rao/. Accessed 17 Aug 2020.

37. RPCEC. Treatment of SARS CoV-2 pneumonia with the antiCD6 monoclonal antibody itolizumab. https://rpcec.sld.cu/ensay os/RPCEC00000311-Sp. Accessed 17 Aug 2020.

38. Livemint, 2020. Biocon to give more data on itolizumab after use in covid-19 treatment rejected. https://www.livemint.com/news/ india/biocon-to-give-more-data-on-itolizumab-after-use-in-covid -19-treatment-rejected-11595858120238.html. Accessed 17 Aug 2020.

39. Science The Wire, 2020. ICMR Says biocon's drug trial not good enough to prove mortality reduction. https://science.thewire.in/ health/icmr-balram-bhargava-biocon-kiran-mazumdar-shaw-itoli zumab-mortality-phase-2-trials/. Accessed 17 Aug 2020.

40. tolizumab (Anti-CD6). CECMED. https://www.cecmed.cu/covid -19/aprobaciones/itolizumab-anti-cd6-0. Accessed 17 Aug 2020.

41. Science The Wire, 2020. How effective are the drugs approved to treat COVID-19 patients in India? https://science.thewire.in/healt h/cdsco-remdesivir-favipiravir-dexamethasone-tocilizumab-itoli zumab/. Accessed 17 Aug 2020.

42. Business today, 2020. India's per-capita income rises $6.8 \%$ to Rs 11,254 a month in FY20. [online] https://www.businesstoday.in/ current/economy-politics/india-per-capita-income-rises-68-tors-11254-a-month-in-fy20/story/393333.html. Accessed 17 Aug 2020. 